\title{
Ultrasound-Guided Erector Spinae Plane Block (US-ESPB) - anaesthetic block: Case Report
}

\author{
Antonio Coviello ${ }^{1}$, Maria Vargas ${ }^{1}$, Gaetano Castellano ${ }^{1}$, Alfredo Maresca ${ }^{1}$, and Giuseppe \\ Servillo ${ }^{1}$
}

${ }^{1}$ Affiliation not available

May 22, 2020

\begin{abstract}
ESP-block produce analgesia. A patient rejects GA for an intervention of capsulated under-apicalular lipoma exeresis. ESPblock is performed. The ESP represents a valid anesthetic alternative for thoracic wall operations whose patients have contraindications to the GA.
\end{abstract}

Ultrasound-Guided Erector Spinae Plane Block(US-ESPB) - anaesthetic block: Case Report Key words

US-ESPB - Erector Spinae Block - ESP - Erector Spinae Plane - Esp Anesthesia.

\section{Key Clinical Message}

ESP block can be a valid alternative to general anesthesia, specially in patients with contraindications to GA - above all in the current Covid 19 pandemy - thanks to the anesthetic mixture diffusion in the epidural space as described by Forero

\section{Introduction}

The ultrasound-guided erector spinae plane block (US-ESPB) at T5 transverse process is recently described as a technique for providing thoracic analgesia [1,2].

From recent literature it is known that the injection into the deep fascial plane to erector spinae muscle (the erector spinae plane, ESP) at the level of the T5 transverse process can produce profound analgesia of the ipsilateral hemithorax [3].

Anatomical dissection indicates that the likely action mechanism is the diffusion of local anaesthetic anteriorly through the connective tissues and ligaments spanning the adjacent transverse processes and into the vicinity of the spinal nerve roots (consistent with other reports of successful analgesia following injection into a similar tissue plane in the thorax $[4,5]$.

ESP is widely used, particularly in thoracic surgery, for intra and postoperative analgesia. Erector Spinae Plane block that determines anaesthesia and not only analgesia has currently little clinical application. This case report shows that in certain cases ESP can also be used as an anaesthetic plan, as an effective and safe alternative to general anaesthesia. Moreover, it did not determine any discomfort of the patient, on the contrary, it improved the surgeons' work.

\section{Case report}


It is here by declared that the patient has consented to the publication of this case report intended purely for scientific and clinical purposes, maintaining the privacy of the processing of her personal data

Our 25-year-old female pz came in our operative block, without comorbidity, to perform an intervention of capsulated under-apicalular lipoma exeresis, under fascial plane, localized in the left hemithorax of about $10 \mathrm{~cm}$ of diameter. The patient reported the story of Awareness during a previous surgery and categorically rejects a second GA. It is therefore decided for the execution of the US-ESPB. With the patient in sitting position, a high-frequency linear probe $(12 \mathrm{MHz})$ was placed in longitudinal orientation at level of the T7 transverse process, $3 \mathrm{~cm}$ from the midline. A 22 gauge $50-\mathrm{mm}$ block needle was inserted in plane, with a cephalad-to-caudad direction (Figure 1), until the tip laid into the plane to deep of the erector spinae muscle. After hydrolocalization with $3 \mathrm{~mL}$ of normal saline to open the plane, $20 \mathrm{~mL}$ of $0.75 \%$ ropivacaine and dexamethasone $8 \mathrm{mg}$ were injected.

A sensory block (Hollmen 4) is obtained with an extension from T5 to L1 - tested with Pin Prick and Ice tests - for the entire duration of the surgery (30') during which the pz was in prone position - in spontaneous breathing - sedated with $0,7 \mathrm{mcg} / \mathrm{kg} / \mathrm{h}$ of dexmedetomidine 10 minutes before the surgery.

The vital parameters were stable throughout the duration of the intervention, on PA 110/70 $\mathrm{mmHg}$, FC 70 bpm, $\mathrm{SpO}_{2} 99 \%$ average.

The pz was collaborating and awakening to verbal stimulus throughout the intervention RASS scale -2

During the post-operative the pz reported Hollmen 3 analgesia at T1 ( $2 \mathrm{~h}$ from the surgery); Hollmen 2 analgesia at T2 (6h from the surgery); Hollmen 1 analgesia at T3 (12h from the surgery).

Together with the full satisfaction of the pz, who reported NRS $<2$ at $24 \mathrm{~h}$ post intervention with no analgesic necessity, even the surgeons were satisfied in proceeding with a constant plan of anaesthesia that did not alter the anatomical planes.

\section{Discussion}

Unlike Forero et al. that showed a large multidermatomic sensory block with the use of minor LA concentrations, our clinical case presented a multidermatomic anesthesiology plan with the use of LA at anesthetic concentrations. As suggested by Forero in his previous reports, it is probably due to the diffusion of the anesthetic solution both in the paravertebral space and in the peridural space.

Moreover US-ESPB has provided a long-lasting analgesia, probably due to the spread of anesthetic solution close to the intervertebral foramina, next to the origin of the dorsal and ventral branches of the thoracic spinal nerves, as suggested by cadaveric investigations.

Further researches, as also suggested by Fusco et al., should be necessary to confirm whether the US-ESPB could be an effective alternative to PVB (paravertebral block).

Paravertebral block (PVB) is currently the gold standard for management of chronic thoracic pain. Unfortunately, contraindications such as coagulopathies or anticoagulant therapies and the difficulty in performing the block, may determine the exclusion of many patients from this treatment.

Chin et al., in their work, have performed a US-ESPB at T7 level in a fresh cadaver and assessed the extent of injectate spread using computerised tomography. There was radiographic evidence of spread extending cranially to the upper thoracic levels and caudally as far as the L2-L3 transverse processes.

Our metameric extension tested with PinPrick and Ice test before surgery can be superimposable about what Chin showed in his work.

ESP-block compared to a LA does not alter the surgical plans and improves the outcome of the patient both in terms of analgesia and surgical times.

The clinical benefits that the US-ESPB has shown must obviously be evaluated on a larger patient court to further ensure the clinical validity demonstrated in this case report. 
In fact, this anaesthetic technique could, in certain clinical cases, replace GA particularly in patients with respiratory and hemodynamic problems.

\section{Conclusion}

The US-ESPB which is mainly used as an antalgic rather than anaesthesiology block, represents a valid anaesthetic alternative for thoracic wall operations whose patients have contraindications to the GA without clinically disrupting the respiratory mechanism and without determining any hemodynamic impact.

In our opinion the direction of the needle is fundamental to address the spread of the anesthetic mixture. In our clinical case the choice of the cranial caudal approach was well considered before performing the procedure. The position of the patient is quite important for the execution of the block. According to our experience the sitting position is the best in terms of simplification of the technique.

\begin{tabular}{lllll}
\hline & Dr. Antonio Coviello 1 & Dr. Maria Vargas 2 & Dr. Gaetano Castellano 3 & Prof. Alfred \\
Concepts & $\mathrm{X}$ & $\mathrm{X}$ & & $\mathrm{X}$ \\
$\begin{array}{l}\text { Definition of intellectual content } \\
\text { Literature search }\end{array}$ & $\mathrm{X}$ & $\mathrm{X}$ & $\mathrm{X}$ & $\mathrm{X}$ \\
$\begin{array}{l}\text { Data acquisition } \\
\text { Data analysis }\end{array}$ & $\mathrm{X}$ & $\mathrm{X}$ & \\
$\begin{array}{l}\text { Manuscript preparation } \\
\text { Manuscript editing }\end{array}$ & $\mathrm{X}$ & $\mathrm{X}$ & $\mathrm{X}$ \\
Manuscript review & $\mathrm{X}$ & $\mathrm{X}$ & & $\mathrm{X}$ \\
\hline
\end{tabular}

Author contribution statement for all authors:

\section{Bibliography}

[1] Forero M, Adhikary SD, Lopex H, Tsui C, Chin KJ. The erector spinae plane block a novel analgesic technique in thoracic neuropathic pain. Regl Anesth Pain Med 2016;41:621-627.

[2] Fusco P, Di Carlo S, Scimia P, Luciani A, Petrucci E, Marinangeli F. Could the new ultrasound-guided erector spinae plane block be a valid alternative to paravertebral block in chronic chest pain syndromes? Minerva Anestesiol. 2017 Oct;83(10):1112-1113

[3] Chin KJ, Adhikary SD, Sarwani N, Forero M. the analgesic efficacy of preoperative bilateral erector spinae plane (ESP) blocks in patients having ventral hernia repair. Anaesthesia . 2017 Apr;72(4):452-460.

[4] Costache I, Sinclair J, Farrash FA, et al. Does paravertebral block require access to the paravertebral space? Anaesthesia2016; 71:858-859.

[5] Rouè C, Wallaert M, Kacha M, Havet E. Intercostal/paraspinal nerve block for thoracic surgery. Anaesthesia 2016; 71:112-113. 


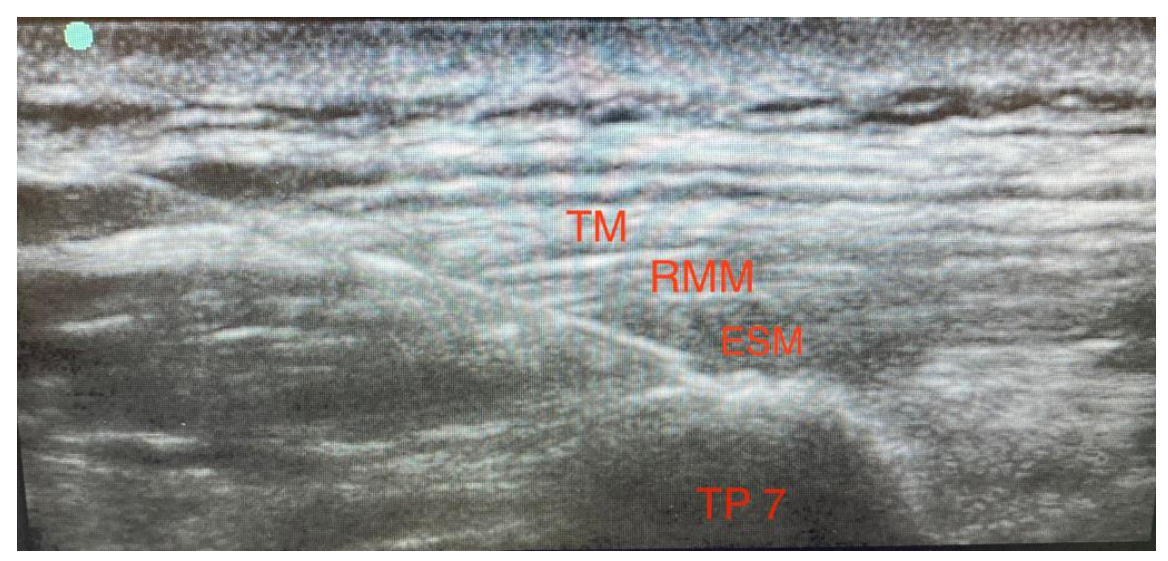

